\title{
Segmental intracellular, interstitial, and intravascular volume changes during simulated hemorrhage and
} resuscitation: A case study \author{
and Marty Loughry ${ }^{4}$ \\ 1. LDM Associates, San Jose, USA \\ 2. Walter Reed Army Institute of Research, Silver Spring, MD, USA \\ Current position: Tulane University School of Medicine, New Orleans, LA, USA \\ 3. New York Medical College, New York, NY, USA \\ 4. UFI, Inc., Morro Bay, USA \\ 5. E- mail any correspondence to:pmontgomery@telis.org
}

Leslie D. Montgomery ${ }^{1,5}$, Richard W. Montgomery ${ }^{1}$, Wayne A. Gerth ${ }^{1}$, Michael Bodo ${ }^{2}$, Julian M. Stewart ${ }^{3}$

\begin{abstract}
This paper describes a new combined impedance plethysmographic (IPG) and electrical bioimpedance spectroscopic (BIS) instrument and software that will allow noninvasive real-time measurement of segmental blood flow, intracellular, interstitial, and intravascular volume changes during various fluid management procedures. The impedance device can be operated either as a fixed frequency IPG for the quantification of segmental blood flow and hemodynamics or as a multi-frequency BIS for the recording of intracellular and extracellular resistances at 40 discrete input frequencies. The extracellular volume is then deconvoluted to obtain its intravascular and interstitial component volumes as functions of elapsed time. The purpose of this paper is to describe this instrumentation and to demonstrate the information that can be obtained by using it to monitor segmental compartment volume responses of a pig model during simulated hemorrhage and resuscitation. Such information may prove valuable in the diagnosis and management of rapid changes in the body fluid balance and various clinical treatments.
\end{abstract}

Keywords: Bioimpedance; compartment volumes; hemorrhage; resuscitation

\section{Introduction}

Shock and hemorrhage are two of the leading causes of death of civilian and military personnel following trauma or injury. Shock is identified with circulatory insufficiency defined by sufficiently severe tissue ischemia that tissue oxygen needs are not met producing oxygen debt, and ultimately tissue damage and multi-organ system failure. The paragon and most studied cause of circulatory failure is hemorrhagic shock in which progressive hypovolemia and reduced numbers of red blood cells jointly decrease oxygen carrying capacity.

While the use of vital signs such as heart rate, blood pressure, temperature, and respirations comprise the hemodynamic quantities classically evaluated in hemorrhagic shock, these quantities mostly reflect the integrated effects of fundamental changes in blood flows and blood volumes that are progressively perturbed during the stages of blood loss. Thus in their classic review of the hemodynamic and neuroendocrine consequences of hemorrhagic hypovolemia, Schadt and Ludbrook [1] refer to the earlier human venesection studies of Barcroft [2] in which rapid and highly controlled hemorrhage occurred in two phases: phase 1 or the sympathoexcitatory phase; and phase 2 or the sympathoinhibitory phase. Together these closely mimic the sequence seen in simple vasovagal faint [3]. As indicated in the figure, total peripheral resistance (TPR), and HR increase, right atrial pressure (RAP) and cardiac output (CO) decrease throughout early (mild) to middle (moderate) 
hemorrhage while blood pressure is relatively well maintained.

Blood pressure is thus the preferentially controlled variable at the expense of $\mathrm{CO}$ so that the largely autoregulated central nervous system (CNS) and heart regional blood flows are maintained. This is an acknowledged oversimplification both from the standpoint of vascular control mechanisms, which involve neurohumoral and local autocrine and paracrine systems, as well as autonomic regulatory mechanisms. Moreover, much more is now understood concerning the effects of hemorrhage on regional blood flow and blood volume distribution. Thus in his seminal work, Vatner [4] described only small increases in mesenteric (splanchnic) and iliac (peripheral vascular) arterial resistances in primates, with overall sustained cardiac output and TPR in mild nonhypotensive hemorrhage. These findings were later well explained by Shoukas [5], Hainsworth [6] and coworkers based on rapid mobilization of venous blood from the splanchnic reservoir in response to carotid sinus baroreceptor function. In Vatner's hands moderate hypotensive hemorrhage was associated with severely increased splanchnic and peripheral vascular resistance and more modestly increased coronary artery resistance. In severe shock arterial resistance is initially globally increased associated with complete splanchnic emptying followed soon after by splanchnic ischemia and complete mobilization of blood reserves prior to complete collapse of peripheral resistance [7].

A few conclusions: First, it appears that hemorrhage (at least under controlled laboratory conditions) progresses in a regular fashion as the result of changes in regional blood flows and blood volumes. Second, one might therefore infer that measuring blood volumes within regional different body segments can be used to monitor the progress of hemorrhage and/or resuscitation.

Recently electrical bioimpedance spectroscopy (BIS) was validated $[8]$ and used $[9,10]$ to monitor fluid shifts between the intracellular, interstitial and intravascular compartments during dialysis. The ZSpec-2, (UFI Inc., Morro Bay, CA) combines two bioimpedance instruments into a single device. It combines a fixed frequency impedance plethysmograph and a multi-frequency electrical bioimpedance spectrograph into one unit.

The IPG mode was used to quantify total segmental conductive volume. The BIS mode was used to monitor segmental intracellular and extracellular compartment volumes as is done by other BIS devices. Our proprietary software was then used to divide the extracellular compartment volume into its intravascular and interstitial components. This same instrumentation was used in this study to demonstrate that it can monitor relative changes in interstitial and intravascular compartment volumes during hemorrhage and subsequent infusion of resuscitation fluids.
Recent work has shown that spectroscopic bioimpedance measurements of the calf can be used to monitor body composition [11], fluid overload [12] and the fluid status of hemodialysis patients $[13,14]$ in humans. These studies concluded that the calf segment is a good place to make such measurements and provide results that reflect whole body fluid changes.

\section{Materials and Methods}

Our hypothesis is that compartment volume changes during hemorrhage and resuscitation can be followed by the use of our ZSpec-2 bioimpedance spectroscopic instrumentation.

A series of pig studies was conducted to demonstrate the ability of the ZSpec- 2 to monitor relative volume and blood flow changes in the calf of pigs and to confirm that the ZSpec- 2 could be used to monitor changes in intracellular, interstitial, and intravascular compartments volumes. Three types of tests (one animal each day) were completed during which the pig was supine and anesthetized:

1) with the electrodes placed on the torso [Figure $1 \mathrm{~A}$ ] to monitor the fluid changes in the torso during simulated hemorrhage,

2) with the ZSpec-2 located on the calf segment [Figure 1B] during simulated hemorrhage to show that the volume changes in the calf are representative of those that take place in the torso, and

3) with the device located on the calf during infusion of Hespan followed by infusion of saline [Figure 1B] to confirm that the ZSpec-2 can differentiate between intravascular volume increases produced by Hespan and increases produced in the intravascular and interstitial compartments during infusion of saline.

\section{Simulated Hemorrhage}

The general approach in this protocol was that pig were hemorrhaged using the Isobaric Wiggers Model $[15,16]$ to 40 $\mathrm{mmHg}$ in 15 minutes. In the Wiggers model, which is a controlled hemorrhage model, the animal is hemorrhaged to a mean arterial pressure of $40 \mathrm{mmHg}$ and typically held there while different physiological phenomena are investigated, then resuscitated. In this protocol, the animal was not held at this pressure for very long before resuscitation started. Once they reached $40 \mathrm{mmHg}$, about $1 / 3$ blood loss, which is $2 \%$ of bodyweight, they were resuscitated to $70 \mathrm{mmHg}$ and held there for 30 minutes. After this time had elapsed, the animals were resuscitated to $90 \mathrm{mmHg}$ and held there for 30 minutes. Shed blood and infused fluids (Hespan and saline) were measured with a balance; its serial port data were recorded. The computer controlled hemorrhage and resuscitation was performed by a MasterFlex pump (Easy Load II, Cole Palmer, Barrington, IL) and M100 resuscitation pump (Infusion Dynamics, Plymouth Meeting, PA) respectively, controlled by an in-house developed computer assisted hemorrhage and resuscitation algorithm. 


\section{Simulated Resuscitation}

Resuscitation was done using colloid or crystalloid solutions, which replaced the lost blood volume, one for one for albumin resuscitation and three for one replacement for crystalloids (i.e. Lactate Ringer). Each animal received a jugular vein catheter for $\mathrm{CO}$ and a femoral artery catheter for pressure monitoring. Another femoral artery catheter was used for blood withdrawal and blood sampling. A femoral venous cannulation was used for resuscitation.

BIS electrodes were regular ECG electrodes. Placement was performed after shaving and cleaning skin with isopropyl alcohol. Placement of the abdominal electrodes are shown in Figure 1A. Electrode positions that were used during the leg measurements are shown in Figure $1 \mathrm{~B}$. Electrodes 1 and 4 in each case are current input sites. Electrodes 2 and 3 are the sampling sites.

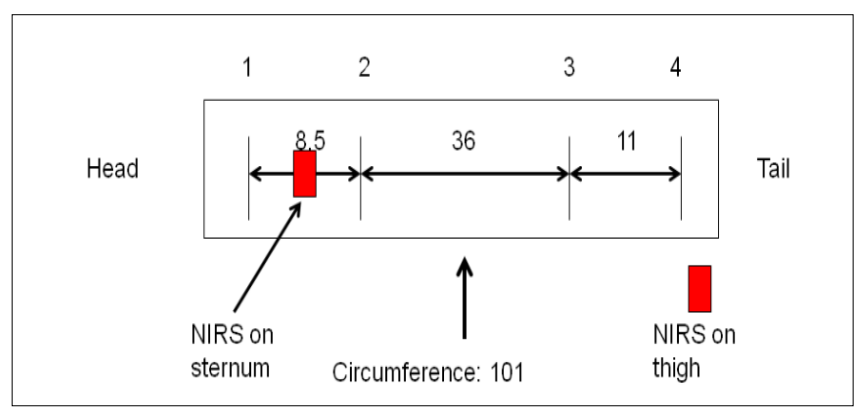

Fig. 1A: Placement of ECG electrodes for abdominal bioimpedance measurements.

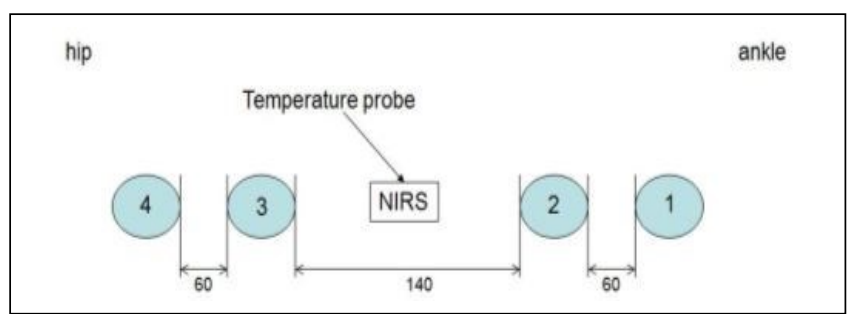

Fig. 1B: Placement of ECG electrodes for leg bioimpedance measurements.

Following the baseline measurements, incremental bleedings were initiated $(30 \%, 45 \%$ and $60 \%$ estimated blood volume). Animals were sacrificed by lethal bleeding.

\section{Data Acquisition and Data Analysis}

Physiological signals were sampled with $200 \mathrm{~Hz}$ analogue-todigital conversion rate using DASH-18 (Astro-Med, West Warwick, RI) and with a Drew computer (Army Institute of Surgical Research, San Antionio, Tx) equipped with an analogue-to-digital converter card (PCl 6052E, National Instruments, Austin, TX); both analogue-to-digital cards had 16 bit resolution. Data were processed off-line. BIS data were recorded by a laptop computer.

\section{Ethical approval}

All animal protocols were submitted to, and approved by, appropriate Animal Use Committees prior to the performance of any animal testing. One animal was tested each day. Each test period lasted between 3-4 hours.

\section{Results and Discussion}

\section{Results of Calf vs. Torso Measurements}

The results of these tests are provided by the time course of changes in two independently measured parameters during simulated hemorrhage. The first, NETFLUID, is the running physical sum of the amount of blood removed (negative values) plus any fluid that was infused positive values) during the period of interest. The other parameter, DVExcell, is the change in extracellular volume (interstitial + blood volumes) calculated from measured BIS data. The time courses of these two parameters during each pig test are shown in Figures 2 and 3.

The NETFLUID profile in each chart is the orchid-colored trace with labels on the left hand Y-axis and the corresponding DVExcell profile is the blue-colored trace with labels on the right hand $\mathrm{Y}$-axis.

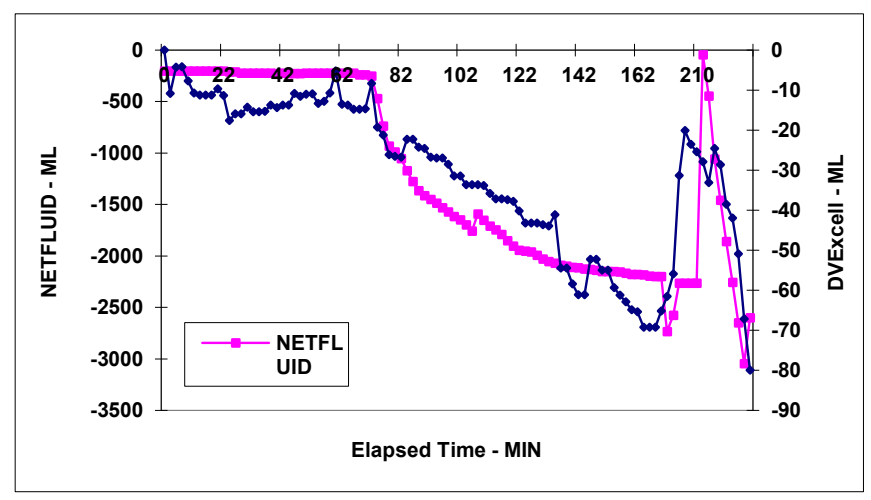

Fig. 2: Changes in NETFLUID and abdominal DVExcell vs elapsed time

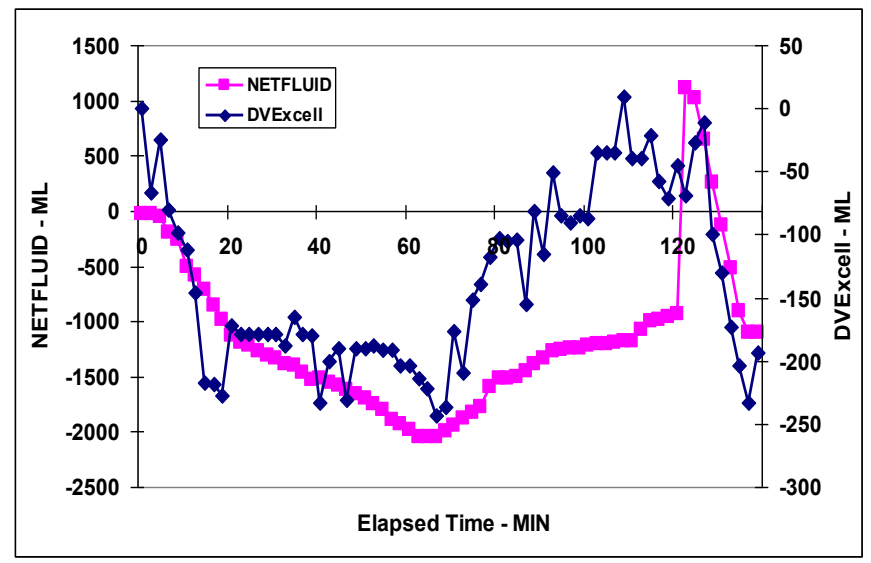

Fig. 3: Changes in NETFLUID and leg DVExcell vs elapsed time.

Figures 2 and 3 show that the ZSpec-2 DVExcell measurements change in a manner that is consistent with NETFLUID. They also indicate that BIS measurements taken on either the abdominal or leg segments can be used to "track" the NETFLUID changes during simulated hemorrhage and resuscitation. 


\section{Results of Simulated Hemorrhage}

The amount of blood withdrawn during the simulated hemorrhage test is shown in Figure 4. The baseline period $(0$ - $90 \mathrm{~min}$ ) is followed sequentially by three bleedings of 500 $\mathrm{ml}$ each at elapsed times of 80, 150 and $210 \mathrm{~min}$.

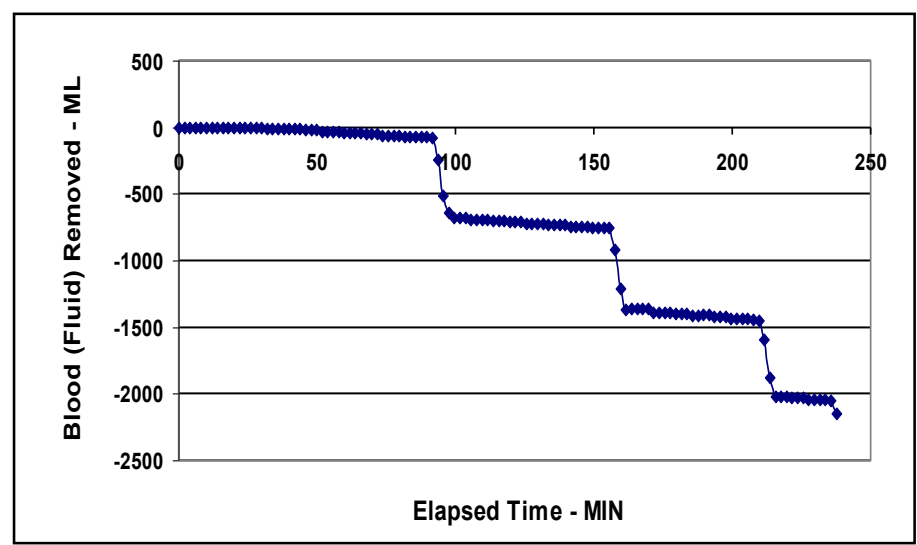

Fig. 4: Volume withdrawn.

The three basal resistance values that were recorded during simulated hemorrhage are shown in Figure 5. Ri and Re (representing the changes in the intracellular and extracellular spaces, respectively) were obtained from the spectroscopic mode. Ro (the total calf resistance that reflects total calf conductive volume) was recorded while the ZSpec2 was operated in the fixed frequency mode at $50 \mathrm{KHz}$. Note that an increase in any of the three resistance values is caused by a reduction in the respective volume. Conversely, a decrease in resistance is due to an increase in the volume being monitored.

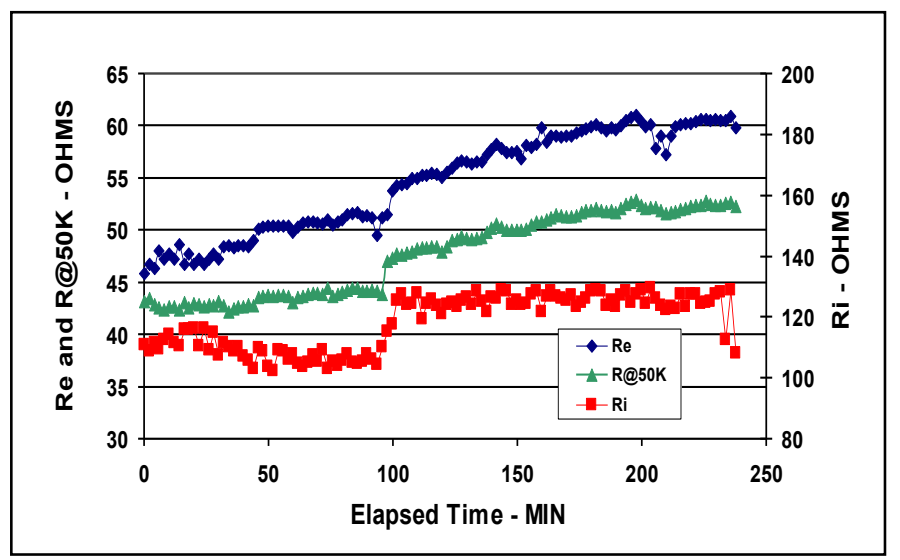

Fig. 5: Basal resistance values.

Fluid is transferred from the intracellular compartment into the extracellular compartment during the initial $0-50 \mathrm{~min}$ of the control period when the pig is placed in a supine position. Both compartment volumes reach an equilibrium after 50 min elapsed time.

During bleeding, Ro, representing the total conductive volume of the calf, is almost identical in relative shape to that of the extracellular compartment resistance, Re. Meanwhile, Ro is quite different in shape from the intracellular resistance, Ri. These similarities/differences may be interpreted as the fact that most of the blood is withdrawn from the extra-cellular space during simulated hemorrhage.

The $\mathrm{Ri}$ and $\mathrm{Re}$ values are then used to compute the intracellular and extracellular compartment volumes of the calf during the simulated hemorrhage. The extracellular volumes is then used to calculate [10] the intravascular and interstitial volumes of the calf. The intravascular (nVblood), intracellular (nVcellular) and interstitial (nVinterstitial) compartment volumes are shown, normalized to the mean of control, in Figure 6.

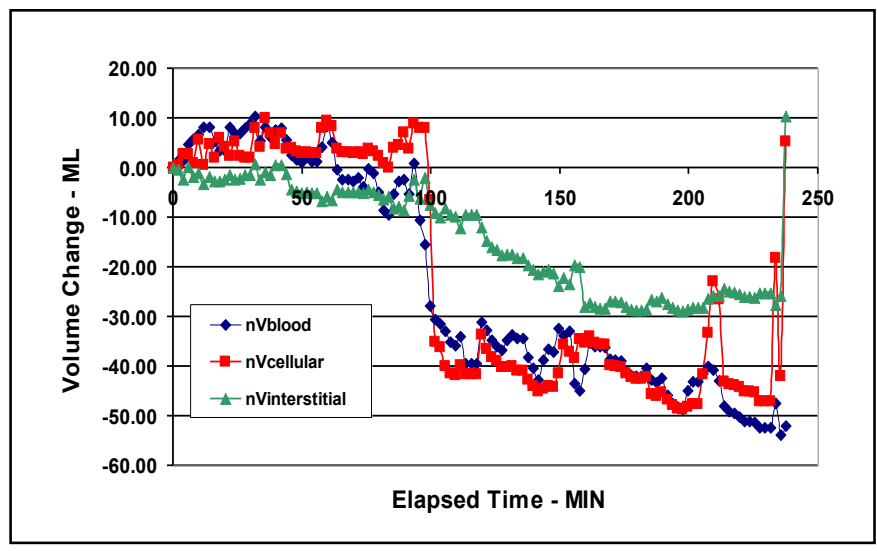

Fig. 6: Compartment volumes.

The volumes in Figure 6 are then differentiated to obtain the fluid transfer rates in and out of each compartment during simulated hemorrhage. Fc is the transfer in or out of the intracellular compartment. $\mathrm{Fi}$ is the similar value of the interstitial compartment. $\mathrm{Fb}$ is that for the intravascular compartment. Initial bleeding causes a large transfer of fluid from the intracellular and intravascular compartments. After the first bleeding most of the remaining fluid is withdrawn from the intravascular compartment.

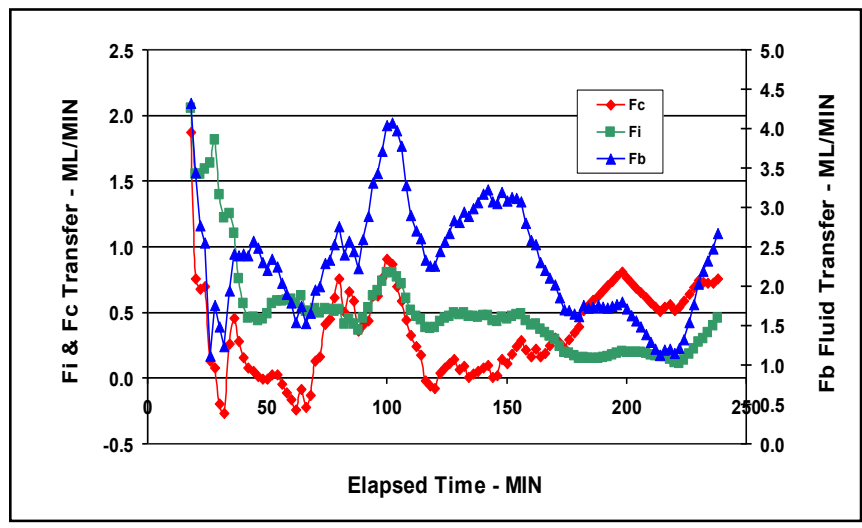

Fig. 7: Fluid transfer between compartment volumes during hemorrhage.

\section{Results of Simulated Resuscitation}

A second pig (Figure 8) was bled twice (starting at 45 min then again at about $110 \mathrm{~min}$ ). It received Hespan following first bleeding at about $100 \mathrm{~min}$ and saline after second bleeding starting at about $175 \mathrm{~min}$. 


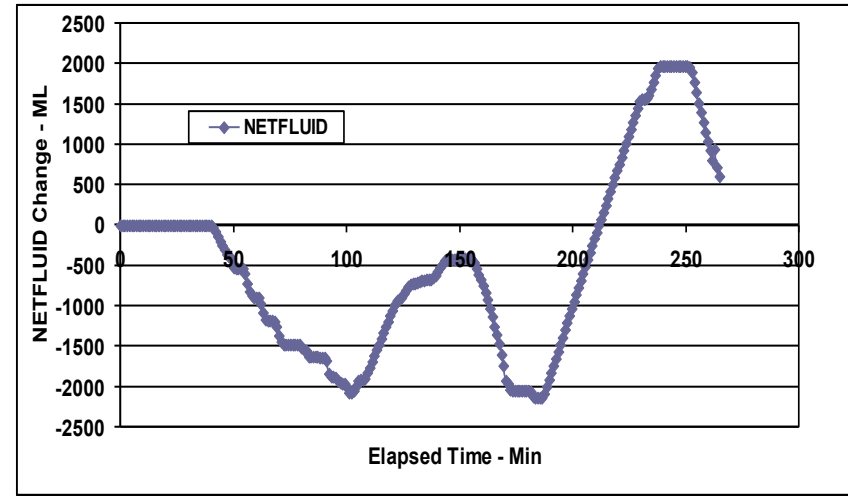

Fig. 8: Resusitation NET FLUID vs. elapsed time.

In Figure 9 are the three basal resistance measurements Re and Ri from the BIS and Ro from the IPG mode. Again, note that the Ri decreases during initial bleeding while Re and Ro increase. Ri seems to reach an equilibrium value and remain fairly constant thereafter. As in the first case, Ro and Re are almost identical in shape. As before, Ro must therefore reflect mostly that the fluid changes during simulated hemorrhage take place in the extracellular compartment.

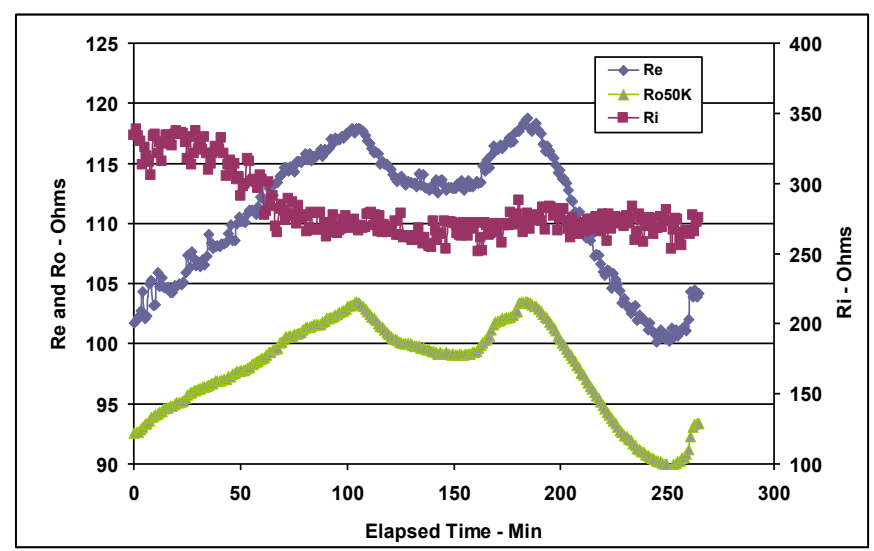

Fig. 9: Basal resistances.

From the BIS data it is possible to compute the volume changes of all three fluid compartments as functions of elapsed time (Figure 10). Again note how the Vinstl decreases during initial bleeding and Vicell increases in volume while Vblood remains fairly steady for about the first 75 min. Fluid must be going from the interstitial compartment into the intracellular space while most of the fluid taken out of the intravascular space is also coming from the interstitial compartment (like refill for dialysis patients).

The effect of the infusion of Hespan and saline on the fluid compartments can be seen in Figure 11. The NETFLUID trace has been added to Figure 11 so that one can see the times of infusion of Hespan (starting about $100 \mathrm{~min}$ ) and then saline starting about $180 \mathrm{~min}$. Note that infusion of Hespan does not affect Vinstl while infusion of saline seems to go into BOTH compartments simultaneously.

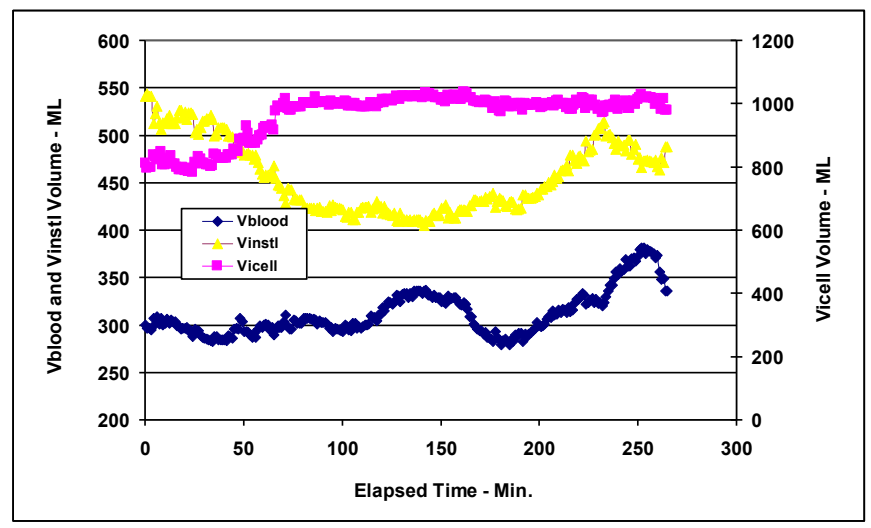

Fig. 10: Compartment volumes vs. elapsed time.

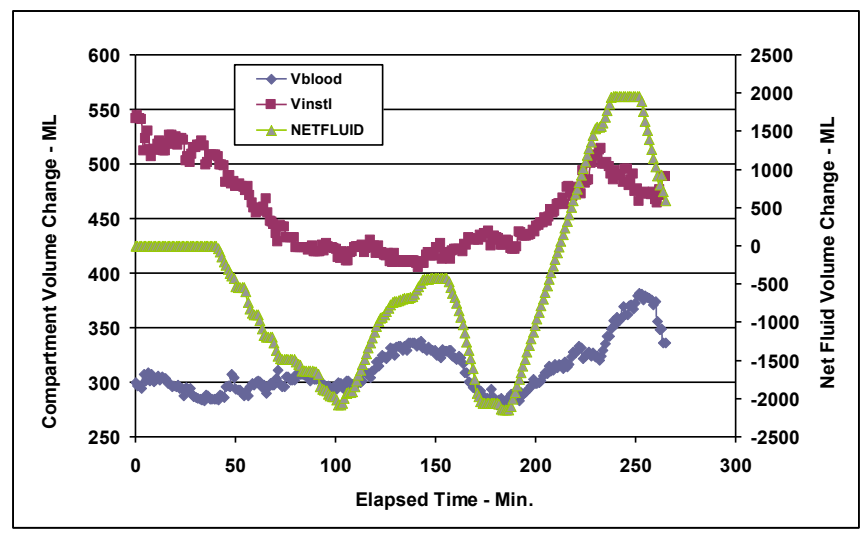

Fig. 11: Intravascular and interstitial compartment volume responses to infusion.

As shown in Figure 11, only Vblood increases during infusion of Hespan and both Vblood and Vinstl increase during infusion of saline. This confirms that the ZSpec-2 can indeed differentiate between changes in the intravascular and interstitial compartments.

The fluid transfer rates between Vinstl and Vblood during the two infusion periods are shown in Figure 12. $\mathrm{Fc}=$ transfer in and out of cells, $\mathrm{Fi}=$ interstitial space, and $\mathrm{Fb}=$ intravascular. Again, you can see that most of the Hespan goes into the vascular system while the saline goes into both the vascular and interstitial space.

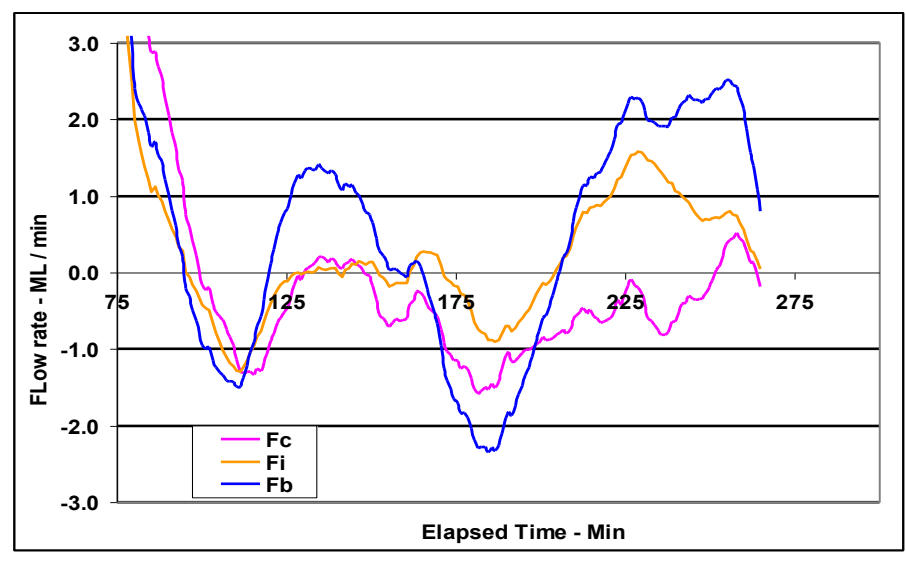

Fig. 12: Compartment fluid transfer rates during infusion. 
Figure 12 illustrates the changes in intravascular, interstitial, and intracellular fluid compartments of the leg following sequential infusion of Hespan and saline. Approximately $80 \%$ of the Hespan, by virtue of the proteins in the fluid, stays within the vascular compartment. Saline on the other hand readily leaks out of the vascular compartment into the interstitial space.

\section{Conclusions}

Figures 2 and 3 show that the ZSpec-2 DVExcell measurements change in a manner that is consistent with NETFLUID. They also indicate that BIS measurements taken on either the abdominal or leg segments can be used to "track" the NETFLUID changes during simulated hemorrhage and resuscitation.

As shown in Figures 10 and 11, only Vblood increases during infusion of Hespan and both Vblood and Vinstl increase during infusion of saline. This confirms that the ZSpec-2 can indeed differentiate between changes in the intravascular and interstitial compartments.

Our ZSpec-2 system permits, for the first time, noninvasive continuous recording and display of fluid volume redistribution between intracellular, interstitial and vascular fluids. There is a critical need for such an instrument as a bedside or operating room monitor in many situations. Here are just a couple examples:

- In critically ill patients who are hypotensive, such as in septic shock, ICU physicians often give large amounts of fluids (as much as 10 or more liters a day) to maintain blood pressure and hemodynamic stability. Ideally, the replacement fluid should stay intravascular to raise blood pressure. Unfortunately, if this fluid moves into the interstitial space, this will lead to massive edema and if it goes into lung tissue, it leads to acute respiratory distress syndrome (ARDS), which is associated with high mortality. Treatment of this complication requires fluid removal by a hemodialysis procedure ("Continuous Veno-Venous Hemofiltration" or (VVH), which is simply removing fluid by ultrafiltration with a hemodialysis filter. Usually these patients require invasive monitoring such as intraarterial placement of central venous catheters to measure wedge pressures, pulmonary arterial pressure, and cardiac output. We think that monitoring extent of interstitial and intracellular volume changes by noninvasive instrument would provide a better guide in fluid management in such patients.

- The ZSpec-2 will also be useful for intravenous fluid management in the acute setting. The on-line monitoring of volume shifts between compartments is crucial particularly in critically ill patients (in septic or cardiogenic shock, burns patients, or perhaps critically patients in the Emergency Room or Operating Room).
Such patients often require large volumes of fluid replacement, resulting in massive fluid overload with variable degrees of interstitial edema (or conceivably cell swelling?). Detection of fluid (water) shifts in the vascular, interstitial and cellular space in a non-invasive manner at the bedside, would be of tremendous help in managing such critically ill patients.

\section{Acknowledgements}

The authors wish to thank Ms. Sharon Hanish and Brian Scholfield of UFI, Inc. Morro Bay, CA for their support in the design, manufacture and implementation of the BIS used in this study. In addition, we wish to acknowledge Drs. Marvin Medow and Frederick J. Pearce for their technical support and encouragement during this project. We acknowledge Steve vanAlbert for his assistance in making our figures.

\section{Conflict of Interest}

None of the authors have received or will receive any compensation or monetary benefit from the publication of this article. This article represents new research and has not been published elsewhere.

\section{Disclosure of Funding}

This work was funded, in part, by the National Institutes of Health through SBIR Grants 1 R43 HL074524-01 and 2 R44 HL074524-02A2 entitled "Intra/Extracellular Volume and Hemodynamics" and, in part, by the U.S. Army Medical Research and Materiel Command D43_0025_2005.

\section{Disclaimer}

Material has been reviewed by the Walter Reed Army Institute of Research. There is no objection to its presentation and/or publication. The opinions or assertions contained herein are the private views of the author, and are not to be construed as official, or as reflecting true views of the Department of the Army or the Department of Defense. Research was conducted under an approved animal use protocol in an AAALAC accredited facility in compliance with the Animal Welfare Act and other federal statutes and regulations relating to animals and experiments involving animals and adheres to principles stated in the Guide for the Care and Use of Laboratory Animals, NRC Publication, 2011 edition.

\section{References}

1. Schadt JC, Ludbrook J. (1991) Hemodynamic and neurohumoral responses to acute hypovolemia in conscious mammals. Am. J Physiol. 260:H305-H318. https://doi.org/10.1152/ajpheart.1991.260.2.h305

2. Barcroft H, McMicahel JE, Sharpey-Schafer EP. (1994) Posthaemorrhagic fainting. Study by cardiac output and forearm flow. Lancet. 1:489-491. https://doi.org/10.1016/s0140-6736(00)74173-0 
3. Julu PO, Cooper VL, Hansen S et al. (2003) Cardiovascular regulation in the period preceding vasovagal syncope in conscious humans. J Physiol. 549:299-311. https://doi.org/10.1113/jphysiol.2002.036715

4. Vatner SF. (1974) Effects of hemorrhage on regional blood flow distribution in dogs and primates. J Clin. Invest. 54:225235. https://doi.org/10.1172/jci107757

5. Shoukas AA, MacAnespie CL, Brunner MJ et al. (1981) The importance of the spleen in blood volume shifts of the systemic vascular bed caused by the carotid sinus baroreceptor reflex in the dog. Circ Res. 49:759-766. https://doi.org/10.1161/01.res.49.3.759

6. Hainsworth R, Karim F. 1974) Changes in abdominal vascular capacity in response to stimulation of efferent sympathetic nerves. J Physiol. 239:26P-27P.

7. Rowell LB. (1993) Human Cardiovascular Control. NY, NY: Oxford University Press.

8. Montgomery LD, Gerth WA, Montgomery RW, Lew SQ, Klein $M D$, Stewart JM, et al. (2013) Monitoring intracellular, interstitial, and intravascular volume changes during fluid management procedures. Med. Biol. Eng. Comput. 51:11671175. https://doi.org/10.1007/s11517-013-1064-3

9. Montgomery LD, Montgomery RW, Gerth WA, Lew SQ, Klein MD, Stewart JM, et al. (2017) Bioimpedance monitoring of cellular hydration during hemodialysis therapy. Hemo. Int. 21(4):575-584. https://doi.org/10.1111/hdi.12511
10. Montgomery LD, Montgomery RW, Gerth WA, Laughry M, Lew SQ, Velasquez MT. (2017) A system to monitor segmental intracellular, interstitial, and intravascular volume and circulatory changes during acute hemodialysis. J. Electr. Bioimp. 8:40-53. https://doi.org/10.5617/jeb.4443

11. Zhu F, Levin NW. (2015) Estimation of body composition and normal fluid status using a calf bioimpedance technique. Blood Purif. 39:25-31. https://doi.org/10.1159/000368937

12. Delano M, Sodini C. (2018) Evaluating calf bioimpedance measurements for fluid overload management in a controlled environment. Physiol. Meas. 39:125009 https://doi.org/-10.1088/1361-6579/aaf277

13. Zhu F, Kotanko P, Handelman GJ, Raimann J, Liu L, et al. (2010) Assessment of degree of hydration in dialysis patients using whole body and calf bioimpedance analysis. J. Physics 224:012124. https://doi. org/10.1088/1742-6596/224/1/012124

14. Liu L, Zhu F, Raimann J, Thijssen S, Sipahioglu MH, et al. (2012) Determination of fluid status in haemodialysis patients with whole body and calf bioimpedance techniques. Nephrology (Carlton) 2: 131-140. https://doi.org/10.1111/j.1440-1797. 2011.01526.x

15. Wiggers C. (1950) Physiology of Shock. New York, Oxford University Press.

16. Bodo M, Montgomery LD, Pearce FJ, Armonda R. (2018) Measurement of cerebral blood flow autoregulation with rheoencephalography: a comparative pig study. J. Electr. Bioimp. 9:123-132. https://doi.org/10.2478/joeb-2018-0017 